\title{
A Case of Miliary Nodules, Hemoptysis and Hot Thyroid Cancer: Unusual Presentation of Papillary Thyroid Cancer
}

\author{
1Jesse SL Hu, ${ }^{2}$ Rajeev Parameswaran
}

\begin{abstract}
Background: Papillary thyroid carcinoma is the commonest thyroid cancer. Patients usually present with thyroid nodule and rarely with hyperthyroidism such that 2009 ATA guidelines recommended that cytological evaluation is not necessary in patients with hyperfunctioning nodules as they rarely harbor malignancy. We report a case of an unusual presentation of metastatic papillary thyroid carcinoma in a young patient.
\end{abstract}

Case presentation: A 17-year-old girl, presented to our hospital with 3 days of fever, cough and hemoptysis. Chest X-ray showed extensive miliary nodules and was treated for presumed miliary tuberculosis. Biochemical investigations revealed a hyperthyroid state (fT4 55.7 TSH <0.02), with negative antibodies (TRAB and TSI). Radioisotope scan showed increased uptake on right lobe. She underwent bronchoscopy and biopsy which revealed metastatic papillary thyroid carcinoma.

Clinical examination revealed a small goiter with palpable cervical node at level III on the left. There were no clinical signs of Graves' disease and she had no history of previous radiation or family history of endocrine disease. Ultrasound revealed multiple hypodense thyroid nodules with microcalcification and increased vascularity. Ultrasound of the neck showed the presence of abnormal lymphadenopathy.

She underwent total thyroidectomy, bilateral central neck dissection and left lateral modified neck dissection. Histology showed $1.3 \mathrm{~cm}$ papillary thyroid carcinoma involving the left lobe and multifocal papillary thyroid microcarcinomas involving both lobes. Ten out of 27 nodes were involved. She was BRAF mutation positive.

She recovered well postoperatively and was rendered hypothyroid. She underwent radioiodine ablation which showed no more disease in the neck but unfortunately there was no uptake in the lung metastases.

Conclusion: Metastatic papillary thyroid cancer developing in a young patient with hyperthyroidism is extremely rare and suggests a more aggressive behavior as confirmed by BRAF mutation.

Keywords: Hyperthyroid, Papillary, Radioiodine, Thyroid cancer.

${ }^{1}$ Associate Consultant, ${ }^{2}$ Assistant Professor and Consultant

${ }^{1}$ Department of Surgery, National University Hospital Singapore

${ }^{2}$ Department of Endocrine Surgery, National University Hospital, Singapore

Corresponding Author: Rajeev Parameswaran, Consultant, Department of Endocrine Surgery, National University Hospital, Singapore, Phone: +6591271524, e-mail: rajeev parameswaran@nuhs.edu.sg
How to cite this article: Hu JSL, Parameswaran R. A Case of Miliary Nodules, Hemoptysis and Hot Thyroid Cancer: Unusual Presentation of Papillary Thyroid Cancer. World J Endoc Surg 2015;7(3):72-75

\section{Source of support: Nil}

Conflict of interest: None

\section{INTRODUCTION}

Papillary thyroid carcinoma is the most common thyroid cancer encountered by any endocrine surgical unit and it is usually associated with good prognosis. The patients commonly present with a thyroid nodule and rarely with hyperthyroidism such that 2009 ATA guidelines recommended that cytological evaluation is not necessary in patients with hyperfunctioning nodules as they rarely harbor malignancy.

Here, we report a case of an unusual presentation of metastatic papillary thyroid carcinoma in a young patient.

\section{CASE REPORT}

A 17-year-old girl who was previously well with no past medical history, presented to our hospital with 3 days of fever, cough and hemoptysis. Initial chest X-ray showed extensive miliary nodules (Fig. 1). Sputum was sent for staining and culture for acid fast bacilli. She was treated for presumed miliary tuberculosis with anti-mycobacterial drugs. During the course of treatment as she was noted to be persistently tachycardic, further blood investigations were performed.

Biochemical investigations revealed a hyperthyroid state (fT4 55.7 TSH <0.02), with negative antibodies (TRAB and TSI). She did not have any clinical or serological features of Graves' disease. Radioisotope scan (Fig. 2) showed slightly increased uptake on right lobe. She was started on carbimazole and propranolol for hyperthyroidism likely secondary to toxic thyroid nodule and discharged well.

Upon further review in clinic, CT scan of the thorax (Figs 3A and B) was performed in view of poor resolution of symptoms and showed multiple military nodules scattered in random distribution in both lungs with the largest nodule measuring up to $3.5 \mathrm{~mm}$. There were prominent left axillary lymph nodes, measuring up to $11 \mathrm{~mm}$. The sputum culture results were found to be negative for mycobacterial infection. She underwent a 
bronchoscopy and biopsy of right middle lobe of lung which revealed metastatic papillary thyroid carcinoma. Immunohistochemical staining was positive for TTF-1, CK-7 and thyroglobulin. She was thus referred to our endocrine surgery unit for further review.

Clinical examination revealed a pleasant girl who was clinically euthyroid and had a small goiter with no obvious nodules. There was a $1 \mathrm{~cm}$ palpable cervical node at level III on the left. There was no history of previous radiation or family history of endocrine disease. Ultrasound and CT scan of the neck revealed multiple thyroid nodules with microcalcification and increased vascularity, with the largest nodule seen at the left thyroid

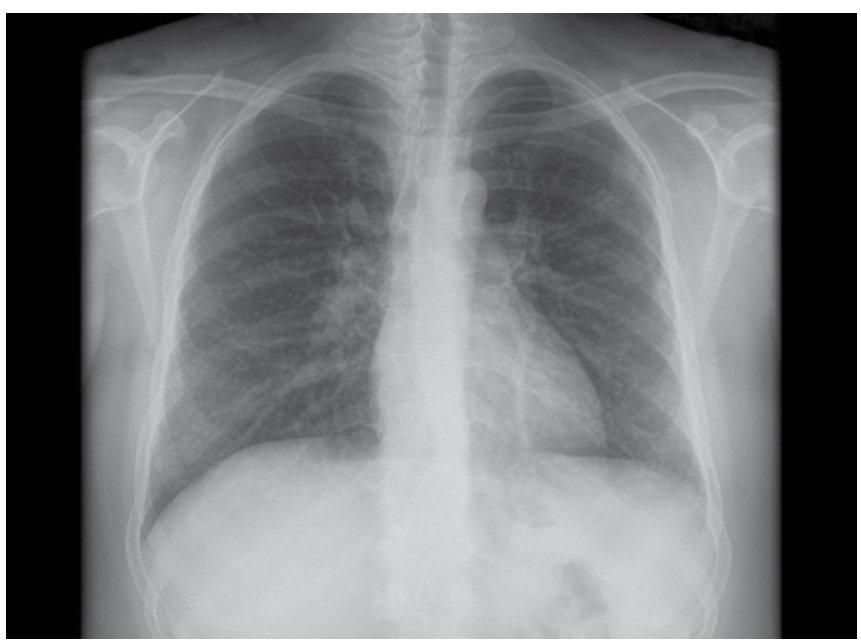

Fig. 1: Chest X-ray showing multiple miliary nodules in the lung fields

RT

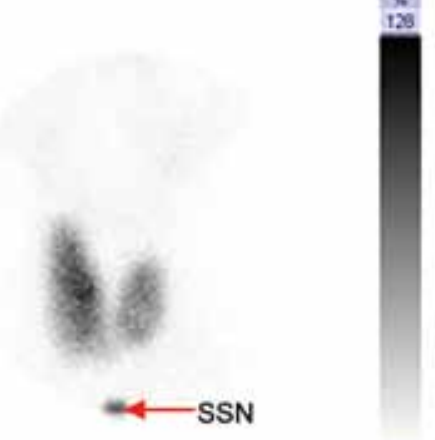

Anterior WMMARKER 100K duration 99 sec.

RT

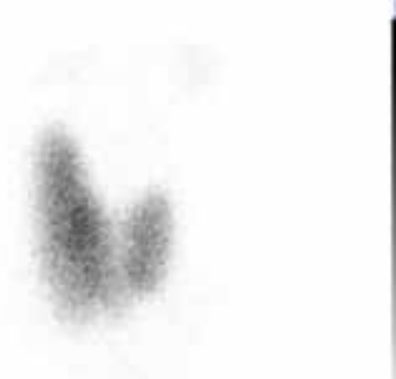

RAO 100K duration: 59 sec. upper pole measuring $15 \mathrm{~mm}$. This was associated with prominent cervical lymph nodes, at levels III, IV, V and VI on the left and level VI on the right. Serum thyroglobulin level was 26.5 and anti-thyroglobulin antibodies were undetectable.

In view of the findings, she was underwent a total thyroidectomy, bilateral central neck dissection and left lateral neck dissection. Histology showed $13 \mathrm{~mm}$ papillary thyroid carcinoma involving the left lobe and multifocal papillary thyroid microcarcinomas involving both lobes. Ten out of 27 lymph nodes were involved. She was also found to be BRAF mutation positive.

She recovered well postoperatively and was rendered hypothyroid. Subsequently, she underwent radioiodine ablation which showed no more disease in the neck. However, despite the proven lung metastases, there was no radioiodine uptake in the lung metastases suggesting that the tumor may have dedifferentiated. This was further confirmed on PET-CT (Fig. 4) which showed varying degrees of FDG uptake compatible with metastases. She was started on thyroxine replacement with the aim for TSH suppression. She continues to well with no further complications, with plans to start tyrosine kinase inhibitors if metastases continue to grow.

\section{DISCUSSION}

Papillary thyroid cancer is the most common thyroid malignancy and clinically manifests as a thyroid nodule, discovered on clinical examination or incidentally on

RT

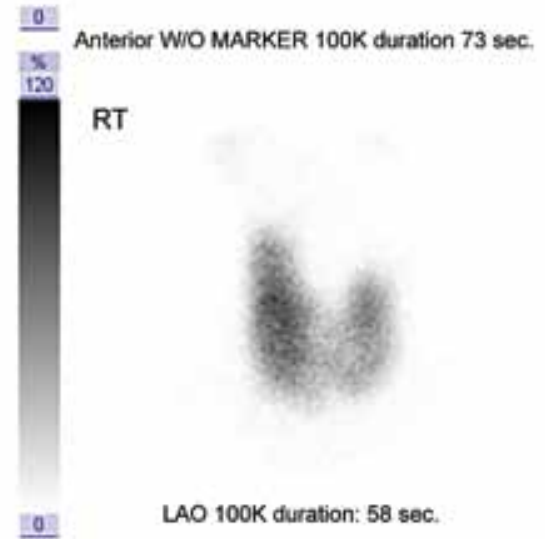

Fig. 2: Radioisotope scan showing a slight increased uptake in a right lobe (SSN: Suprasternal notch) 

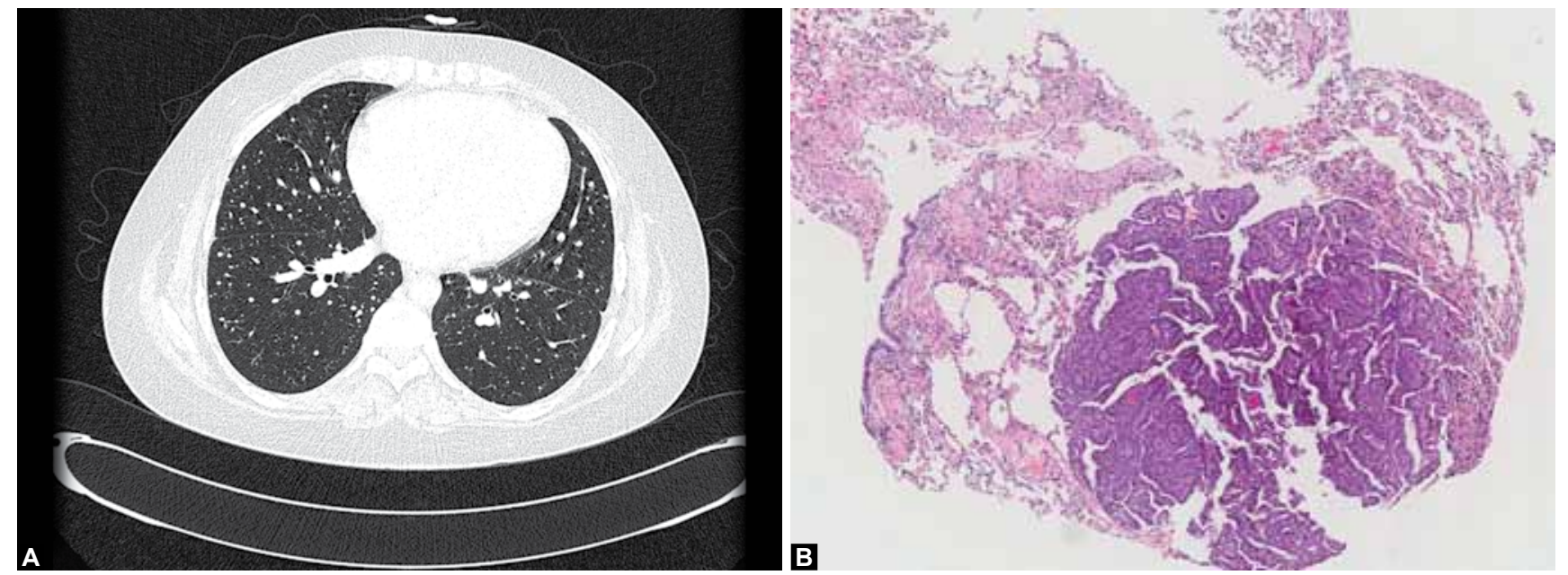

Figs $3 A$ and B: (A) Computed tomography thorax showing multiple miliary nodules in keeping with metastasis and (B) histology of transbronchial lung biopsy showing nodule of metastatic papillary thyroid carcinoma (Courtesy: Dr Seet JE, Department of Pathology, NUHS)

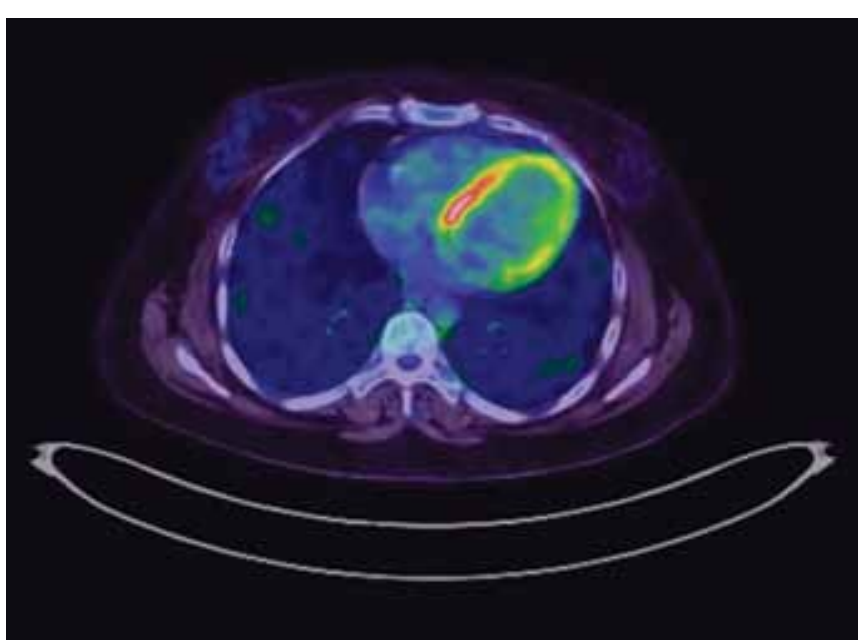

Fig. 4: Positron emission tomography CT showing lung metastases

imaging. It is not uncommon to diagnose cancers following total thyroidectomy for either Graves' disease or nodular goiter. ${ }^{1-3}$ However, for thyroid cancer to present with hyperthyroidism in the absence of diagnosis of Graves' disease or toxic nodular goiter is uncommon, as is the case with our patient. Even more uncommon is the fact that the patient presented with hemoptysis secondary to the extensive bilateral lung metastases instead of the primary.

Historically, it was believed that hyperthyroid state protects against thyroid malignancy ${ }^{4}$ and the incidence of cancers reported in patients undergoing surgery for Graves' disease was up to $2.5 \% .{ }^{5,6}$ However, more recent series have shown the rates to be much higher at 1.6 to $21 \% .^{7}$ A recent 4 -year population-based cohort study showed an increased risk of thyroid cancer (6.8 fold over patients with normothyroid state) and the risk increased with duration of hyperthyroidism. ${ }^{8}$ The difference in outcomes of thyroid cancers in patients who present with hyperthyroid states in comparison to the euthyroid states is poorly studied.
Patients with hyperthyroid state are investigated with a radioiodine uptake scan and in the presence of cold nodules, needs further imaging with an ultrasound scan along with a needle biopsy. The use of ultrasound in Graves' disease may lead to early detection of nodules not felt by clinical examination ${ }^{9}$ and characterize the nodules as malignant in the presence of echogenicity, ill-defined borders, absence of a peripheral hypoechoic rim, no cystic changes, increased intranodular blood flow, and punctate calcifications ${ }^{10,11}$ as seen in our patient.

Distant metastasis is seen in 1 to $7 \%$ of patients with differentiated thyroid cancer, with lung being the most common site followed by bone. ${ }^{12-14}$ The patients present with hemoptysis or dyspnea at rest or upon exertion in the presence of lung metastasis. The radioiodine avid lesions in the lungs are usually treated with radioactive iodine (RAI) ablative therapy, but in some cases it may be difficult to completely eradicate with radioiodine therapy. ${ }^{15}$ In absence of effective ablation with radioiodine therapy of lung metastasis one has to consider the possibility of dedifferentiation.

This case highlights the importance of carefully evaluating patients with hyperthyroid state in the presence of thyroid nodules. The earlier belief that 'hot nodules' are unlikely to be malignant is no more true. To conclude, the detection of the rare truly hot thyroid carcinomas remains a clinical challenge.

\section{REFERENCES}

1. Cakir M,AriciC,AlakusH,AltunbasH,BalciMK,KarayalcinU. Incidental thyroid carcinoma in thyrotoxic patients treated by surgery. Hormone Research 2007;67(2):96-99.

2. Chen YK, Lin CL, Chang YJ, Cheng FT, Peng CL, Sung FC, et al. Cancer risk in patients with Graves' disease: a nationwide cohort study. Thyroid: Official J Am Thyroid Assoc 2013;23(7): 879-884. 
3. Gabriele R, Letizia C, Borghese M, De Toma G, Celi M, Izzo L, et al. Thyroid cancer in patients with hyperthyroidism. Hormone Res 2003;60(2):79-83.

4. Means JH. Present day trends in thyroid research. Western J Surg Obstet Gynecol 1948;56(2):65-71.

5. Sokal JE. Incidence of malignancy in toxic and nontoxic nodular goiter. J Am Med Assoc 1954;154(16):1321-1325.

6. Beahrs OH, J PJ, Black BM. Nodular goiter and malignant lesions of the thyroid gland. J Clin Endocrinol Metabol 1951; 11(10):1157-1165.

7. Pazaitou-Panayiotou K, Michalakis K, Paschke R. Thyroid cancer in patients with hyperthyroidism. Hormone and Metabolic Research 2012;44(4):255-262.

8. Yeh NC, Chou CW, Weng SF, Yang CY, Yen FC, Lee SY, et al. Hyperthyroidism and thyroid cancer risk: a population-based cohort study. Experimental and clinical endocrinology and diabetes: Official Journal, German Society of Endocrinology [and] German Diabetes Association 2013;121(7):402-406.

9. Kim WB, Han SM, Kim TY, Nam-Goong IS, Gong G, Lee HK, et al. Ultrasonographic screening for detection of thyroid cancer in patients with Graves' disease. Clin Endocrinol 2004;60(6):719-725.

10. Papini E, Guglielmi R, Bianchini A, Crescenzi A, Taccogna S, Nardi F, et al. Risk of malignancy in nonpalpable thyroid nodules: predictive value of ultrasound and color-Doppler features. J Clin Endocrinol Metabol 2002;87(5):1941-1946.

11. Berker D, IsikS, Ozuguz U, Tutuncu YA, Kucukler K, Akbaba G, et al. Prevalence of incidental thyroid cancer and its ultrasonographic features in subcentimeter thyroid nodules of patients with hyperthyroidism. Endocrine 2011;39(1):13-20.

12. Massin JP, Savoie JC, Garnier H, Guiraudon G, Leger FA, Bacourt F. Pulmonary metastases in differentiated thyroid carcinoma. Study of 58 cases with implications for the primary tumor treatment. Cancer 1984;53(4):982-992.

13. Ruegemer JJ, Hay ID, Bergstralh EJ, Ryan JJ, Offord KP, Gorman CA. Distant metastases in differentiated thyroid carcinoma: a multivariate analysis of prognostic variables. J Clinical Endocrinol Metabol 1988;67(3):501-508.

14. Schlumberger M, Tubiana M, De Vathaire F, Hill C, Gardet $P$, Travagli JP, et al. Long-term results of treatment of 283 patients with lung and bone metastases from differentiated thyroid carcinoma. J Clin Endocrinol Metabol 1986;63(4): 960-967.

15. Sisson JC, Jamadar DA, Kazerooni EA, Giordano TJ, Carey JE, Spaulding SA. Treatment of micronodular lung metastases of papillary thyroid cancer: are the tumors too small for effective irradiation from radioiodine? Thyroid: Official J Am Thyroid Assoc 1998;8(3):215-221. 